\title{
Synthesis of 8-(5-Aryl-4-Octyl-2-Phenyl-3, 4-Dihydro-2H-Pyrazol- 3-yl)-Octanoic acid ethyl esters via 1, 3-Dipolar Cycloaddition Reaction
}

\author{
${ }^{1}$ M. Govindaraju, ${ }^{2}$ G. Vasanth Kumar, ${ }^{3}$ B.N. Mylarappa, ${ }^{4}$ K. Ajay Kumar* \\ 1,2,4, Department of Chemistry, Yuvaraja's College, University of Mysore, Mysore, India. \\ ${ }^{3}$ Transplant surgery section, Rangos Research Center, University of Pittsburgh, PA 15201, USA.
}

\begin{abstract}
Aldehyde phenyl hydrazones 2 a-i undergo oxidative dehydrogenation with Chloramine-T to give nitrile imines, which are trapped in situ by ethyl oleate 1 to afford 8-(5-Aryl-4-octyl-2-phenyl-3,4-dihydro-2Hpyrazol-3-yl)-octanoic acid ethyl esters 3a-i in good yield. The structures of the cycloadducts were confirmed by spectral studies and elemental analysis.
\end{abstract}

Key words: Pyrazoles, pyrazolines, chloramine-T, 1,3-dipolar cycloaddition, cycloadducts.

\section{Introduction}

These heterocyclic compounds containing pyrazole nucleus have flourished with considerable intensity because of their synthetic and biological applications. The most convenient synthesis of pyrazole ring system has been executed in the literature via 1,3-dipolar cycloaddition reactions of alkenes and alkynes with nitrile imines generated in situ from aldehyde phenylhydrazones. The literature review shows that pyrazoles have known to exhibit enormous biological activity such as antimicrobial [1-2], antioxidant [3], antiviral [4], antitubercular [5], antimicobacterial [6] antitumor and antiangiogenic agents [7]. A series of structurally related $1 H$-pyrazolyl derivatives synthesized compounds were tested for their anti-inflammatory and antimicrobial activities. In addition, COX-1 and COX-2 inhibitory activities, ulcerogenic effects and acute toxicity were determined [8]. The synthesis of 1-Aryl-3[nitro-2-thienyl]-4-aroyl pyrazoles have been reported by the 1,3dipolar cycloaddition of 3-arylsydnones with 1-aryl-3-[5-nitro2-thienyl]-2-propyn-1-ones and were screened for antibacterial and antifungal activity [9].

The usual synthesis of nitrile imines involves the thermolysis or photolysis of tetrazole [10], oxidation of aldehyde hydrazones with lead tetraacetate [11]. Rai and co-workers [12] reported a new approach for the synthesis of pyrazoles via 1,3-Diplar cycloaddition of acetyl acetone and in situ generated nitrile imines by the catalytic dehydrogenation of phenylhydrazone using chloramine- $T$ as oxidant and they obtained the regioselective cycloadducts. Later they demonstrated the successful synthesis and evaluation of pyrazole derivatives for their antimicrobial and antioxidant activity [13-14]. This paper describes the successful synthesis of new title compounds via 1.3-dipolar cycloaddition reactions of in situ generated nitrile imines with ethyl oleate as dipolarophile.

\section{Materials And Methods}

The chemicals/reagents used were purchased from sigma-aldrich chemicals (India) and Merck Chemicals (India). IR spectra were recorded on a Nujol mull on Shimadzu 8300 spectrometer. The ${ }^{1} \mathrm{H}$ NMR and ${ }^{13} \mathrm{C}$ NMR spectra were recorded on a Bruker supercon $400 \mathrm{MHz}$ spectrophotometer using $\mathrm{CDCl}_{3}$ as solvent and TMS as an internal standard. The Chemical shifts are expressed in $\delta \mathrm{ppm}$. Mass spectra were obtained on Shimadzu LCMS-2010A spectrophotometer (chemical ionization) and the important fragments are given with the relative intensities in the bracket. Elemental analysis was obtained on a Thermo Finnigan Flash EA 1112 CHN analyser. Thin layer chromatography (TLC) were performed on a pre-coated Silica Gel sheets (HF 254, sd-fine) using benzene:ethyl acetate (7:2) eluent and visualization of the spots was done in iodine vapour and UV light. Chromatographic separations were carried out on silica gel (70-230 mesh, Merck) column using hexane:ethyl acetate $(8: 1)$ as eluent.

In a typical intermolecular 1,3-dipolar cycloaddition reaction, the nitrile imines generated by the catalytic dehydrogenation of hydrazones $\mathbf{2}$ using chloramine-T as oxidizing agent were trapped in situ by ethyl oleate $\mathbf{1}$. After the completion of the reaction and usual work up; the reaction afforded 8-(4-Octyl-5-aryl-2-phenyl-3,4dihydro-2H-pyrazol-3-yl)-octanoic acid ethyl esters (5) in moderate to good yield (Scheme-1). 


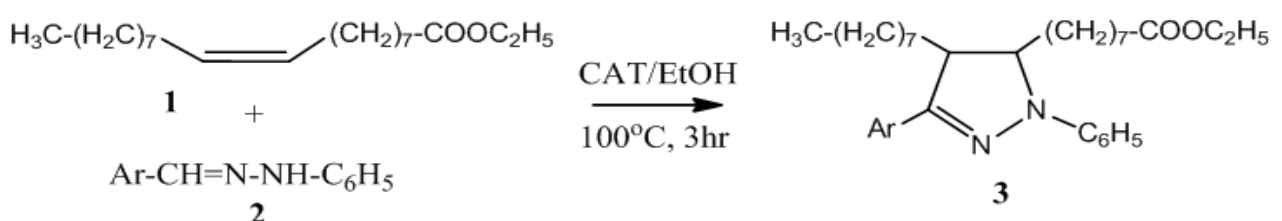
3 a) $\mathrm{Ar}=\mathrm{C}_{6} \mathrm{H}_{5}$;
b) $\mathrm{Ar}=4-\mathrm{OCH}_{3} \mathrm{C}_{6} \mathrm{H}_{4}$;
c) $\mathrm{Ar}=3,4-\left(\mathrm{OCH}_{3}\right)_{2} \mathrm{C}_{6} \mathrm{H}_{3} ;$ d) $\mathrm{Ar}=4-\mathrm{CH}_{3} \mathrm{C}_{6} \mathrm{H}_{4}$;
e) $\mathrm{Ar}=4-\mathrm{FC}_{6} \mathrm{H}_{4} ;$ f) $\mathrm{Ar}=4-\mathrm{ClC}_{6} \mathrm{H}_{4}$;
g) $\mathrm{Ar}=4-\mathrm{BrC}_{6} \mathrm{H}_{4}$;
h) $\mathrm{Ar}=4-\mathrm{NO}_{2} \mathrm{C}_{6} \mathrm{H}_{4}$;

i) $\mathrm{Ar}=$ Furan-2-oyl.

Scheme 1

\section{Results And Discussion}

The general synthetic pathway employed is depicted in the scheme-1. The structures of the cycloadducts were provided by IR, ${ }^{1} \mathrm{H}$ NMR, ${ }^{13} \mathrm{C}$ NMR, MS studies and elemental analysis. For instance in IR Spectra, the peak expected due to $=\mathrm{N}-\mathrm{NH}$ - group in the region $3300-3150 \mathrm{~cm}^{-1}$ of the starting material hydrazone was found absent and all shows peaks in the region $3300-3150 \mathrm{~cm}^{-1}$ due to $=\mathrm{N}-\mathrm{N}$ - stretching. Further, they showed ester carbonyl stretching at $1710-1730 \mathrm{~cm}^{-1}$. In ${ }^{1} \mathrm{H}$ NMR spectra, the signals due to $\mathrm{C}_{3}-\mathrm{H}$ appears as doublet of doublet in the region $\delta 3.9-4.2 \mathrm{ppm}$ and the signals due to $\mathrm{C}_{4}-\mathrm{H}$ appears as doublet of doublet in the region $\delta$ 5.1-5.4 ppm. The coupling constant $(J)$ values calculated for $\mathrm{C}_{3}-\mathrm{H}$ and $\mathrm{C}_{4}-\mathrm{H}$ were found in range 7.0-9.6 $\mathrm{Hz}$, these values suggests that both $\mathrm{C}_{3}-\mathrm{H}$ and $\mathrm{C}_{4}-\mathrm{H}$ are cis orientation and the cycloaddition took place in cis fashion. Apart from these all showed the signals due to aromatic and substituent protons in the expected region, which favors the formation of cycloadducts.

In ${ }^{13} \mathrm{C}$ NMR spectra, the signals due to $\mathrm{C}_{3}$-carbon appear in the region $\delta 51.0-54.0 \mathrm{ppm}$. the signals due to $\mathrm{C}_{4}$-carbon appear in the region $\delta 42.0-44.0 \mathrm{ppm}$. And the signals due to $\mathrm{C}_{5}$-carbon appear in the region $\delta 42.0$ $44.0 \mathrm{ppm}$. All showed the signals due to aromatic carbons and substituent carbons in the expected region. All the cycloadducts gave significantly stable molecular ion peaks with a relative abundance ranging from 20-56\% and base peak at m/e 311(M-substituted phenyl nitrile, benzyne and $\mathrm{CH}_{2}$ ). Further, all showed satisfactorily elemental analysis with a deviation of $\pm 0.02 \%$ from the theoretically calculated values. These observations strongly favor the formation of the cycloadducts $3 \mathrm{a}-\mathrm{i}$.

\section{Experimental}

General procedure for the synthesis of 8-(5-Aryl-4-octyl-2-phenyl-3,4-dihydro-2H-pyrazol-3-yl)octanoic acid ethyl esters (3): A mixture of aldehyde phenylhydrazone 2 (5 mmol), ethyl oleate 1 (5 mmol) and chloramine-T trihydrate $(5.5 \mathrm{mmol})$ was refluxed on a water bath for 3-4 hours. The progress of the reaction was monitored by TLC. After completion of the reaction, the salts formed were filtered off; the solvent was evaporated in vacuum. The residual mass was extracted into ether $(1 \times 25 \mathrm{~mL})$, washed successively with water $(3 \times 20 \mathrm{~mL}), 5 \%$ sodium hydroxide $(2 \times 10 \mathrm{~mL})$, brine solution $(1 \times 15 \mathrm{~mL})$ and dried over anhydrous sodium sulphate. Evaporation of the solvent afforded crude oily substance $\mathbf{3}$, which was purified by column chromatography using benzene: ethyl acetate $(6: 1)$ as eluent. The same procedure was used in all cases.

4.1 Synthesis of 8-(4-Octyl-2,5-diphenyl-3,4-dihydro-2H-pyrazol-3-yl)-octanoic acid ethyl ester (3a): Obtained from benzaldehyde phenylhydrazone $\mathbf{2 a}(5 \mathrm{mmol})$, ethyl oleate $\mathbf{1}(5 \mathrm{mmol})$ and chloramine-T trihydrate $(5.5 \mathrm{mmol})$ as an oil $62 \%$ yield. $\quad{ }^{1} \mathrm{H} \mathrm{NMR} \mathrm{CDCl}_{3}: \delta 0.97\left(\mathrm{t}, 3 \mathrm{H}, \mathrm{CH}_{3}\right), 1.26\left(\mathrm{~s}, 18 \mathrm{H}, \mathrm{CH}_{2}\right), 1.30(\mathrm{t}$, $\left.3 \mathrm{H}, \mathrm{CH}_{3}\right), 1.32\left(\mathrm{~s}, 2 \mathrm{H}, \mathrm{CH}_{2}\right), 1.34\left(\mathrm{~s}, 2 \mathrm{H}, \mathrm{CH}_{2}\right), 1.48\left(\mathrm{~s}, 2 \mathrm{H}, \mathrm{CH}_{2}\right), 1.62\left(\mathrm{~s}, 2 \mathrm{H}, \mathrm{CH}_{2}\right), 2.26\left(\mathrm{~s}, 2 \mathrm{H}, \mathrm{CH}_{2}\right), 3.96$ $\left(\mathrm{dd}, 1 \mathrm{H}, \mathrm{C}_{3}-\mathrm{H}, J=8.6 \mathrm{~Hz}\right), 4.12\left(\mathrm{~s}, 2 \mathrm{H}, \mathrm{CH}_{2}\right), 5.32\left(\mathrm{dd}, 1 \mathrm{H}, \mathrm{C}_{4}-\mathrm{H}, J=8.2 \mathrm{~Hz}\right), 6.42\left(\mathrm{~s}, 2 \mathrm{H}, \mathrm{C}_{6} \mathrm{H}_{5}-\mathrm{H}\right), 6.56(\mathrm{~s}, 1 \mathrm{H}$, $\left.\mathrm{C}_{6} \mathrm{H}_{5}-\mathrm{H}\right), 7.02\left(\mathrm{~s}, 2 \mathrm{H}, \mathrm{C}_{6} \mathrm{H}_{5}-\mathrm{H}\right), 7.32(\mathrm{~s}, 3 \mathrm{H}, \mathrm{Ar}-\mathrm{H}), 7.62(\mathrm{~s}, 2 \mathrm{H}, \mathrm{Ar}-\mathrm{H}) .{ }^{13} \mathrm{C} \mathrm{NMR} \mathrm{CDCl}_{3}: \delta 13.6\left(1 \mathrm{C}, \mathrm{CH}_{3}\right), 14.0$ (1C, $\left.\underline{\mathrm{CH}}_{3}\right), 23.2\left(1 \mathrm{C}, \underline{\mathrm{CH}}_{2}\right), 23.8\left(1 \mathrm{C}, \underline{\mathrm{CH}}_{2}\right), 25.2\left(1 \mathrm{C}, \underline{\mathrm{C}} \underline{H}_{2}\right), 25.6\left(1 \mathrm{C}, \underline{\mathrm{CH}}_{2}\right), 28.2\left(1 \mathrm{C}, \underline{\mathrm{CH}}_{2}\right), 30.0\left(3 \mathrm{C}, \underline{\mathrm{CH}}_{2}\right)$, 30.4 (3C, $\left.\underline{\mathrm{CH}}_{2}\right), 32.4\left(1 \mathrm{C}, \underline{\mathrm{CH}}_{2}\right), 33.8\left(1 \mathrm{C}, \underline{\mathrm{CH}}_{2}\right), 34.4\left(1 \mathrm{C}, \underline{\mathrm{CH}}_{2}\right), 43.3\left(1 \mathrm{C}, \underline{\mathrm{C}}_{4}\right), 52.8\left(1 \mathrm{C}, \underline{\mathrm{C}}_{3}\right), 59.4\left(1 \mathrm{C}, \mathrm{O}^{\mathrm{C}} \mathrm{H}_{2}\right)$, $113.4\left(2 \mathrm{C}, \mathrm{C}_{6} \mathrm{H}_{5}-\mathrm{C}\right), 116.8\left(1 \mathrm{C}, \mathrm{C}_{6} \mathrm{H}_{5}-\mathrm{C}\right), 128.2(2 \mathrm{C}, \mathrm{Ar}-\mathrm{C}), 128.8(2 \mathrm{C}, \mathrm{Ar}-\underline{\mathrm{C}}), 129.3\left(2 \mathrm{C}, \mathrm{C}_{6} \mathrm{H}_{5}-\mathrm{C}\right), 130.6(1 \mathrm{C}$, Ar- $\underline{\mathrm{C}}), 131.8$ (1C, Ar- $\underline{\mathrm{C}}), 143.5$ (1C, $\left.\mathrm{C}_{6} \mathrm{H}_{5}-\underline{\mathrm{C}}\right), 155.8$ (1C, $\left.\underline{\mathrm{C}}_{5}\right), 172.2$ (1C, $\left.\underline{\mathrm{C}}=\mathrm{O}\right) . \mathrm{MS}$ (relative abundance) $\mathrm{m} / \mathrm{z}$ : 505( $\mathrm{MH}^{+}$, 24), 311 (100), 297 (34), 265 (52), 248 (22). Anal. Calcd. for $\mathrm{C}_{33} \mathrm{H}_{48} \mathrm{~N}_{2} \mathrm{O}_{2} ; \mathrm{C}, 78.53 ; \mathrm{H}, 9.59 ; \mathrm{N}$, $5.55 \%$; Found: C, 78.50; H, 9.52; N, 5.48\%.

4.2 Synthesis of 8-[5-(4-Methoxyphenyl)-4-octyl-2-phenyl-3,4-dihydro-2H-pyrazol-3-yl]-octanoic acid ethyl ester (3b): Obtained from 4-Methoxybenzaldehyde phenylhydrazone $\mathbf{2 b}$ (5 mmol), ethyl oleate 1 (5 mmol) and chloramine-T trihydrate $(5.5 \mathrm{mmol})$ in ethyl alcohol $(20 \mathrm{~mL})$ as thick oil in $51 \%$ yield. ${ }^{1} \mathrm{H} \mathrm{NMR} \mathrm{CDCl}_{3}: \delta 0.95$ $\left(\mathrm{t}, 3 \mathrm{H}, \mathrm{CH}_{3}\right), 1.24\left(\mathrm{~s}, 18 \mathrm{H}, \mathrm{CH}_{2}\right), 1.32\left(\mathrm{t}, 3 \mathrm{H}, \mathrm{CH}_{3}\right), 1.34\left(\mathrm{~s}, 2 \mathrm{H}, \mathrm{CH}_{2}\right), 1.36\left(\mathrm{~s}, 2 \mathrm{H}, \mathrm{CH}_{2}\right), 1.46\left(\mathrm{~s}, 2 \mathrm{H}, \mathrm{CH}_{2}\right), 1.60$ $\left(\mathrm{s}, 2 \mathrm{H}, \mathrm{CH}_{2}\right), 2.28\left(\mathrm{~s}, 2 \mathrm{H}, \mathrm{CH}_{2}\right), 3.76\left(\mathrm{~s}, 3 \mathrm{H}, \mathrm{OCH}_{3}\right), 4.00\left(\mathrm{dd}, 1 \mathrm{H}, \mathrm{C}_{3}-\mathrm{H}, J=8.6 \mathrm{~Hz}\right), 4.10\left(\mathrm{~s}, 2 \mathrm{H}, \mathrm{CH}_{2}\right), 5.26$ $\left(\mathrm{dd}, 1 \mathrm{H}, \mathrm{C}_{4}-\mathrm{H}, J=8.2 \mathrm{~Hz}\right), 6.44\left(\mathrm{~s}, 2 \mathrm{H}, \mathrm{C}_{6} \mathrm{H}_{5}-\mathrm{H}\right), 6.59\left(\mathrm{~s}, 1 \mathrm{H}, \mathrm{C}_{6} \mathrm{H}_{5}-\mathrm{H}\right), 6.90(\mathrm{~s}, 2 \mathrm{H}, \mathrm{Ar}-\mathrm{H}), 7.06\left(\mathrm{~s}, 2 \mathrm{H}, \mathrm{C}_{6} \mathrm{H}_{5}-\mathrm{H}\right)$, $7.52(\mathrm{~s}, 2 \mathrm{H}, \mathrm{Ar}-\mathrm{H}) .{ }^{13} \mathrm{C}$ NMR $\mathrm{CDCl}_{3}: \delta 13.8\left(1 \mathrm{C}, \underline{\mathrm{CH}}_{3}\right), 14.1\left(1 \mathrm{C}, \underline{\mathrm{CH}_{3}}\right), 23.6\left(1 \mathrm{C}, \underline{\mathrm{CH}}_{2}\right), 23.9\left(1 \mathrm{C}, \underline{\mathrm{CH}}_{2}\right), 25.1$ (1C, $\left.\underline{\mathrm{CH}}_{2}\right), 25.7\left(1 \mathrm{C}, \underline{\mathrm{CH}}_{2}\right), 28.0\left(1 \mathrm{C}, \underline{\mathrm{CH}}_{2}\right), 30.2\left(3 \mathrm{C}, \underline{\mathrm{CH}}_{2}\right), 30.6\left(3 \mathrm{C}, \underline{\mathrm{CH}}_{2}\right), 32.2\left(1 \mathrm{C}, \underline{\mathrm{CH}}_{2}\right), 33.9\left(1 \mathrm{C}, \underline{\mathrm{CH}}_{2}\right)$, 
Synthesis of 8-(5-Aryl-4-Octyl-2-Phenyl-3,4-Dihydro-2H-Pyrazol-3-yl)-Octanoic acid ethyl esters Via $34.6\left(1 \mathrm{C}, \underline{\mathrm{CH}}_{2}\right), 43.8\left(1 \mathrm{C}, \underline{\mathrm{C}}_{4}\right), 52.8\left(1 \mathrm{C}, \underline{\mathrm{C}}_{3}\right), 56.1\left(1 \mathrm{C}, \mathrm{O}_{\mathrm{CH}}\right), 59.5\left(1 \mathrm{C}, \mathrm{OCH}_{2}\right), 113.2\left(2 \mathrm{C}, \mathrm{C}_{6} \mathrm{H}_{5}-\underline{\mathrm{C}}\right), 114.6$ (2C, Ar- $\underline{\mathrm{C}}), 116.6\left(1 \mathrm{C}, \mathrm{C}_{6} \mathrm{H}_{5}-\underline{\mathrm{C}}\right), 123.7$ (1C, Ar- $\left.\underline{\mathrm{C}}\right), 129.4\left(2 \mathrm{C}, \mathrm{C}_{6} \mathrm{H}_{5}-\underline{\mathrm{C}}\right), 130.2(2 \mathrm{C}, \mathrm{Ar}-\underline{\mathrm{C}}), 143.4\left(1 \mathrm{C}, \mathrm{C}_{6} \mathrm{H}_{5}-\underline{\mathrm{C}}\right)$, $155.4\left(1 \mathrm{C}, \underline{\mathrm{C}}_{5}\right), 164.3(1 \mathrm{C}, \mathrm{Ar}-\underline{\mathrm{C}}), 172.8(1 \mathrm{C}, \underline{\mathrm{C}}=\mathrm{O})$. MS (relative abundance) $\mathrm{m} / \mathrm{z}: 535\left(\mathrm{MH}^{+}, 20\right), 311(100)$, 297 (28), 265 (46), 248 (18). Anal. Calcd. For $\mathrm{C}_{34} \mathrm{H}_{50} \mathrm{~N}_{2} \mathrm{O}_{3}$; C, 76.36; H, 9.42; N, 5.24\%; Found: C, 76.53; H, $9.39 ; \mathrm{N}, 5.15 \%$.

4.3 Synthesis of 8-[5-(3,4-Dimethoxyphenyl)-4-octyl-2-phenyl-3,4-dihydro-2H-pyrazol-3-yl]-octanoic acid ethyl ester (3c): Obtained from 3,4-Dimethoxybenzaldehyde phenylhydrazone 32c ( $5 \mathrm{mmol}$ ), ethyl oleate 1 (5 $\mathrm{mmol})$ and chloramine-T trihydrate $(5.5 \mathrm{mmol})$ in ethyl alcohol $(20 \mathrm{~mL})$ as brown oil in $55 \%$ yield. ${ }^{1} \mathrm{H}$ NMR $\mathrm{CDCl}_{3}: \delta 0.94\left(\mathrm{t}, 3 \mathrm{H}, \mathrm{CH}_{3}\right), 1.20\left(\mathrm{~s}, 18 \mathrm{H}, \mathrm{CH}_{2}\right), 1.28\left(\mathrm{t}, 3 \mathrm{H}, \mathrm{CH}_{3}\right), 1.32\left(\mathrm{~s}, 2 \mathrm{H}, \mathrm{CH}_{2}\right), 1.35\left(\mathrm{~s}, 2 \mathrm{H}, \mathrm{CH}_{2}\right), 1.44(\mathrm{~s}$, $\left.2 \mathrm{H}, \mathrm{CH}_{2}\right), 1.58\left(\mathrm{~s}, 2 \mathrm{H}, \mathrm{CH}_{2}\right), 2.26\left(\mathrm{~s}, 2 \mathrm{H}, \mathrm{CH}_{2}\right), 3.74\left(\mathrm{~s}, 6 \mathrm{H}, \mathrm{OCH}_{3}\right), 4.14\left(\mathrm{~s}, 2 \mathrm{H}, \mathrm{CH}_{2}\right), 4.20\left(\mathrm{dd}, 1 \mathrm{H}, \mathrm{C}_{3}-\mathrm{H}\right.$, $J=8.6 \mathrm{~Hz}$ ), $5.30\left(\mathrm{dd}, 1 \mathrm{H}, \mathrm{C}_{4}-\mathrm{H}, J=8.2 \mathrm{~Hz}\right), 6.48\left(\mathrm{~s}, 2 \mathrm{H}, \mathrm{C}_{6} \mathrm{H}_{5}-\mathrm{H}\right), 6.66\left(\mathrm{~s}, 1 \mathrm{H}, \mathrm{C}_{6} \mathrm{H}_{5}-\mathrm{H}\right), 7.08\left(\mathrm{~s}, 2 \mathrm{H}, \mathrm{C}_{6} \mathrm{H}_{5}-\mathrm{H}\right)$, $6.74(\mathrm{~s}, 1 \mathrm{H}, \mathrm{Ar}-\mathrm{H}), 7.08(\mathrm{~s}, 1 \mathrm{H}, \mathrm{Ar}-\mathrm{H}), 7.14(\mathrm{~s}, 1 \mathrm{H}, \mathrm{Ar}-\mathrm{H}) .{ }^{13} \mathrm{C} \mathrm{NMR} \mathrm{CDCl}_{3}: \delta 13.4\left(1 \mathrm{C}, \underline{\mathrm{CH}}_{3}\right), 14.8\left(1 \mathrm{C}, \underline{\mathrm{CH}}_{3}\right)$, $23.2\left(1 \mathrm{C}, \underline{\mathrm{C}} \mathrm{H}_{2}\right), 23.6\left(1 \mathrm{C}, \underline{\mathrm{C}} \mathrm{H}_{2}\right), 25.4\left(1 \mathrm{C}, \underline{\mathrm{CH}}_{2}\right), 25.9\left(1 \mathrm{C}, \underline{\mathrm{C}} \mathrm{H}_{2}\right), 28.1\left(1 \mathrm{C}, \underline{\mathrm{C}} \mathrm{H}_{2}\right), 30.1\left(3 \mathrm{C}, \underline{\mathrm{CH}}_{2}\right), 30.6(3 \mathrm{C}$, $\left.\underline{\mathrm{CH}}_{2}\right), 32.3\left(1 \mathrm{C}, \underline{\mathrm{CH}}_{2}\right), 33.8\left(1 \mathrm{C}, \underline{\mathrm{CH}}_{2}\right), 34.5\left(1 \mathrm{C}, \underline{\mathrm{CH}}_{2}\right), 44.0\left(1 \mathrm{C}, \underline{\mathrm{C}}_{4}\right), 53.2\left(1 \mathrm{C}, \underline{\mathrm{C}}_{3}\right), 56.5\left(2 \mathrm{C}, \mathrm{OCH}_{3}\right), 59.8(1 \mathrm{C}$, $\left.\mathrm{O}_{\mathrm{CH}_{2}}\right), 113.8\left(2 \mathrm{C}, \mathrm{C}_{6} \mathrm{H}_{5}-\underline{\mathrm{C}}\right), 115.2(1 \mathrm{C}, \mathrm{Ar}-\underline{\mathrm{C}}), 115.8(1 \mathrm{C}, \mathrm{Ar}-\underline{\mathrm{C}}), 116.4\left(1 \mathrm{C}, \mathrm{C}_{6} \mathrm{H}_{5}-\underline{\mathrm{C}}\right), 122.4(1 \mathrm{C}, \mathrm{Ar}-\underline{\mathrm{C}}), 124.2$ $(1 \mathrm{C}, \mathrm{Ar}-\underline{\mathrm{C}}), 129.0\left(2 \mathrm{C}, \mathrm{C}_{6} \mathrm{H}_{5}-\underline{\mathrm{C}}\right), 143.8\left(1 \mathrm{C}, \mathrm{C}_{6} \mathrm{H}_{5}-\underline{\mathrm{C}}\right), 147.3$ (1C, Ar-C), 149.6 (1C, $\left.\mathrm{Ar}-\mathrm{C}\right), 156.2\left(1 \mathrm{C}, \mathrm{C}_{5}\right), 173.0$ (1C, $\underline{\mathrm{C}}=\mathrm{O}$ ). MS (relative abundance) $\mathrm{m} / \mathrm{z}: 565\left(\mathrm{MH}^{+}, 20\right), 311$ (100), 297 (36), 265 (40), 248 (23). Anal. Calcd. For $\mathrm{C}_{35} \mathrm{H}_{52} \mathrm{~N}_{2} \mathrm{O}_{4} ; \mathrm{C}, 74.43 ; \mathrm{H}, 9.28 ; \mathrm{N}, 4.96 \%$; Found: C, 74.33; H, 9.19; N, 4.85\%.

4.4 Synthesis of 8-[5-(4-Methylphenyl)-4-octyl-2-phenyl-3,4-dihydro-2H-pyrazol-3-yl]-octanoic acid ethyl ester (3d): Obtained from 4-Methylbenzaldehyde phenylhydrazone $\mathbf{2 d}(5 \mathrm{mmol})$, ethyl oleate 1 (5 mmol) and chloramine-T trihydrate $(5.5 \mathrm{mmol})$ in ethyl alcohol $(20 \mathrm{~mL})$ as an oil in $68 \%$ yield. ${ }^{1} \mathrm{H} \mathrm{NMR} \mathrm{CDCl}: \delta 0.96(\mathrm{t}$, $\left.3 \mathrm{H}, \mathrm{CH}_{3}\right), 1.06$ (s, $\left.3 \mathrm{H}, \mathrm{CH}_{3}\right), 1.26$ (s, $\left.18 \mathrm{H}, \mathrm{CH}_{2}\right), 1.30\left(\mathrm{t}, 3 \mathrm{H}, \mathrm{CH}_{3}\right), 1.36\left(\mathrm{~s}, 2 \mathrm{H}, \mathrm{CH}_{2}\right), 1.38\left(\mathrm{~s}, 2 \mathrm{H}, \mathrm{CH}_{2}\right), 1.46$ (s, $\left.2 \mathrm{H}, \mathrm{CH}_{2}\right), 1.62\left(\mathrm{~s}, 2 \mathrm{H}, \mathrm{CH}_{2}\right), 2.29\left(\mathrm{~s}, 2 \mathrm{H}, \mathrm{CH}_{2}\right), 4.02\left(\mathrm{dd}, 1 \mathrm{H}, \mathrm{C}_{3}-\mathrm{H}, J=8.6 \mathrm{~Hz}\right), 4.14\left(\mathrm{~s}, 2 \mathrm{H}, \mathrm{CH}_{2}\right), 5.28(\mathrm{dd}, 1 \mathrm{H}$, $\left.\mathrm{C}_{4}-\mathrm{H}, J=8.2 \mathrm{~Hz}\right), 6.42\left(\mathrm{~s}, 2 \mathrm{H}, \mathrm{C}_{6} \mathrm{H}_{5}-\mathrm{H}\right), 6.56\left(\mathrm{~s}, 1 \mathrm{H}, \mathrm{C}_{6} \mathrm{H}_{5}-\mathrm{H}\right), 6.90(\mathrm{~s}, 2 \mathrm{H}, \mathrm{Ar}-\mathrm{H}), 7.08\left(\mathrm{~s}, 2 \mathrm{H}, \mathrm{C}_{6} \mathrm{H}_{5}-\mathrm{H}\right), 7.50(\mathrm{~s}$, $2 \mathrm{H}, \mathrm{Ar}-\mathrm{H})$. Anal. Calcd. For $\mathrm{C}_{34} \mathrm{H}_{50} \mathrm{~N}_{2} \mathrm{O}_{2} ; \mathrm{C}, 78.72 ; \mathrm{H}, 9.72 ; \mathrm{N}, 5.40 \%$; Found: C, 78.63; H, 9.65; N, 5.35\%.

4.5 Synthesis of 8-[5-(4-Fluorophenyl)-4-octyl-2-phenyl-3,4-dihydro-2H-pyrazol-3-yl]-octanoic acid ethyl ester (3e): Obtained from 4-Fluorobenzaldehyde phenylhydrazone $2 \mathbf{e}(5 \mathrm{mmol})$, ethyl oleate 1 (5 mmol) and chloramine-T trihydrate $(5.5 \mathrm{mmol})$ in ethyl alcohol $(20 \mathrm{~mL})$ as brown oil in $48 \%$ yield. ${ }^{1} \mathrm{H} \mathrm{NMR} \mathrm{CDCl}_{3}: \delta 0.97$ (t, $\left.3 \mathrm{H}, \mathrm{CH}_{3}\right), 1.22\left(\mathrm{~s}, 18 \mathrm{H}, \mathrm{CH}_{2}\right), 1.26\left(\mathrm{t}, 3 \mathrm{H}, \mathrm{CH}_{3}\right), 1.30\left(\mathrm{~s}, 2 \mathrm{H}, \mathrm{CH}_{2}\right), 1.36\left(\mathrm{~s}, 2 \mathrm{H}, \mathrm{CH}_{2}\right), 1.46\left(\mathrm{~s}, 2 \mathrm{H}, \mathrm{CH}_{2}\right), 1.62$ $\left(\mathrm{s}, 2 \mathrm{H}, \mathrm{CH}_{2}\right), 2.29\left(\mathrm{~s}, 2 \mathrm{H}, \mathrm{CH}_{2}\right), 4.18\left(\mathrm{dd}, 1 \mathrm{H}, \mathrm{C}_{3}-\mathrm{H}, J=8.6 \mathrm{~Hz}\right), 4.29\left(\mathrm{~s}, 2 \mathrm{H}, \mathrm{CH}_{2}\right), 5.16\left(\mathrm{dd}, 1 \mathrm{H}, \mathrm{C}_{4}-\mathrm{H}, J=8.2\right.$ $H z), 6.50\left(\mathrm{~s}, 2 \mathrm{H}, \mathrm{C}_{6} \mathrm{H}_{5}-\mathrm{H}\right), 6.62\left(\mathrm{~s}, 1 \mathrm{H}, \mathrm{C}_{6} \mathrm{H}_{5}-\mathrm{H}\right), 7.06\left(\mathrm{~s}, 2 \mathrm{H}, \mathrm{C}_{6} \mathrm{H}_{5}-\mathrm{H}\right), 7.12(\mathrm{~s}, 2 \mathrm{H}, \mathrm{Ar}-\mathrm{H}), 7.58(\mathrm{~s}, 2 \mathrm{H}, \mathrm{Ar}-\mathrm{H})$. ${ }^{13} \mathrm{C} \mathrm{NMR} \mathrm{CDCl}_{3}: \delta 13.8\left(1 \mathrm{C}, \underline{\mathrm{CH}}_{3}\right), 14.8\left(1 \mathrm{C}, \underline{\mathrm{CH}}_{3}\right), 23.2\left(1 \mathrm{C}, \underline{\mathrm{CH}}_{2}\right), 24.4\left(1 \mathrm{C}, \underline{\mathrm{CH}}_{2}\right), 25.5\left(1 \mathrm{C}, \underline{\mathrm{CH}}_{2}\right), 26.7(1 \mathrm{C}$, $\left.\underline{\mathrm{CH}}_{2}\right), 28.3\left(1 \mathrm{C}, \underline{\mathrm{CH}}_{2}\right), 30.6\left(3 \mathrm{C}, \underline{\mathrm{C}} \mathrm{H}_{2}\right), 31.0\left(3 \mathrm{C}, \underline{\mathrm{CH}}_{2}\right), 32.7\left(1 \mathrm{C}, \underline{\mathrm{CH}}_{2}\right), 33.9\left(1 \mathrm{C}, \underline{\mathrm{C}} \mathrm{H}_{2}\right), 34.8\left(1 \mathrm{C}, \underline{\mathrm{CH}}_{2}\right), 44.5$ $\left(1 \mathrm{C}, \underline{\mathrm{C}}_{4}\right), 53.8\left(1 \mathrm{C}, \underline{\mathrm{C}}_{3}\right), 59.9\left(1 \mathrm{C}, \mathrm{OCH}_{2}\right), 113.3\left(2 \mathrm{C}, \mathrm{C}_{6} \mathrm{H}_{5}-\underline{\mathrm{C}}\right), 115.4(2 \mathrm{C}, \mathrm{Ar}-\underline{\mathrm{C}}), 116.0\left(1 \mathrm{C}, \mathrm{C}_{6} \mathrm{H}_{5}-\underline{\mathrm{C}}\right), 126.6$ (1C, Ar- $\underline{\mathrm{C}}), 129.0$ (2C, $\left.\mathrm{C}_{6} \mathrm{H}_{5}-\underline{\mathrm{C}}\right), 130.6(2 \mathrm{C}, \mathrm{Ar}-\underline{\mathrm{C}}), 143.3$ (1C, $\left.\mathrm{C}_{6} \mathrm{H}_{5}-\underline{\mathrm{C}}\right), 156.1$ (1C, $\left.\underline{\mathrm{C}}_{5}\right), 165.0$ (1C, Ar- $\left.\underline{\mathrm{C}}\right)$, 173.9 (1C, $\underline{\mathrm{C}}=\mathrm{O}$ ). MS (relative abundance) m/z: 523( $\left.\mathrm{MH}^{+}, 20\right), 311$ (100), 297 (26), 265 (55), 248 (28). Anal. Calcd. For $\mathrm{C}_{33} \mathrm{H}_{47} \mathrm{FN}_{2} \mathrm{O}_{2} ; \mathrm{C}, 75.82 ; \mathrm{H}, 9.06 ; \mathrm{N}, 5.36 \%$; Found: C, 78.73; H, 9.00; N, 5.25\%.

4.6 Synthesis of 8-[5-(4-Chlorophenyl)-4-octyl-2-phenyl-3,4-dihydro-2H-pyrazol-3-yl]-octanoic acid ethyl ester (3f): Obtained from 4-Chlorobenzaldehyde phenylhydrazone $2 \mathbf{f}(5 \mathrm{mmol})$, ethyl oleate 1 (5 mmol) and chloramine-T trihydrate $(5.5 \mathrm{mmol})$ in ethanol $(20 \mathrm{~mL})$ as thick oil in $58 \%$ yield. ${ }^{1} \mathrm{H}$ NMR $\mathrm{CDCl}_{3}: \delta 0.98(\mathrm{t}$, $\left.3 \mathrm{H}, \mathrm{CH}_{3}\right), 1.22$ (s, $\left.18 \mathrm{H}, \mathrm{CH}_{2}\right), 1.29\left(\mathrm{t}, 3 \mathrm{H}, \mathrm{CH}_{3}\right), 1.34\left(\mathrm{~s}, 2 \mathrm{H}, \mathrm{CH}_{2}\right), 1.38\left(\mathrm{~s}, 2 \mathrm{H}, \mathrm{CH}_{2}\right), 1.46\left(\mathrm{~s}, 2 \mathrm{H}, \mathrm{CH}_{2}\right), 1.64$ (s, $\left.2 \mathrm{H}, \mathrm{CH}_{2}\right), 2.28\left(\mathrm{~s}, 2 \mathrm{H}, \mathrm{CH}_{2}\right), 4.08\left(\mathrm{dd}, 1 \mathrm{H}, \mathrm{C}_{3}-\mathrm{H}, J=8.6 \mathrm{~Hz}\right), 4.18\left(\mathrm{~s}, 2 \mathrm{H}, \mathrm{CH}_{2}\right), 5.28\left(\mathrm{dd}, 1 \mathrm{H}, \mathrm{C}_{4}-\mathrm{H}, J=8.2 \mathrm{~Hz}\right)$, $6.54\left(\mathrm{~s}, 2 \mathrm{H}, \mathrm{C}_{6} \mathrm{H}_{5}-\mathrm{H}\right), 6.68\left(\mathrm{~s}, 1 \mathrm{H}, \mathrm{C}_{6} \mathrm{H}_{5}-\mathrm{H}\right), 7.08\left(\mathrm{~s}, 2 \mathrm{H}, \mathrm{C}_{6} \mathrm{H}_{5}-\mathrm{H}\right), 7.28(\mathrm{~s}, 2 \mathrm{H}, \mathrm{Ar}-\mathrm{H}), 7.56(\mathrm{~s}, 2 \mathrm{H}, \mathrm{Ar}-\mathrm{H}) .{ }^{13} \mathrm{C}$ NMR $\mathrm{CDCl}_{3}: \delta 13.1\left(1 \mathrm{C}, \underline{\mathrm{CH}}_{3}\right), 14.7\left(1 \mathrm{C}, \underline{\mathrm{CH}}_{3}\right), 23.0\left(1 \mathrm{C}, \underline{\mathrm{CH}}_{2}\right), 23.8\left(1 \mathrm{C}, \underline{\mathrm{CH}}_{2}\right), 25.6\left(1 \mathrm{C}, \underline{\mathrm{CH}}_{2}\right), 26.1(1 \mathrm{C}$, $\left.\underline{\mathrm{C}} \mathrm{H}_{2}\right), 28.0\left(1 \mathrm{C}, \underline{\mathrm{CH}}_{2}\right), 30.2\left(3 \mathrm{C}, \underline{\mathrm{CH}}_{2}\right), 30.7\left(3 \mathrm{C}, \underline{\mathrm{CH}}_{2}\right), 32.6\left(1 \mathrm{C}, \underline{\mathrm{CH}}_{2}\right), 33.9\left(1 \mathrm{C}, \underline{\mathrm{CH}}_{2}\right), 34.6\left(1 \mathrm{C}, \underline{\mathrm{CH}}_{2}\right), 44.4$ $\left(1 \mathrm{C}, \mathrm{C}_{4}\right), 53.6\left(1 \mathrm{C}, \mathrm{C}_{3}\right), 59.2\left(1 \mathrm{C}, \mathrm{OCH}_{2}\right), 113.4\left(2 \mathrm{C}, \mathrm{C}_{6} \mathrm{H}_{5}-\mathrm{C}\right), 116.8\left(1 \mathrm{C}, \mathrm{C}_{6} \mathrm{H}_{5}-\mathrm{C}\right), 129.0(1 \mathrm{C}, \mathrm{Ar}-\mathrm{C}), 129.5$ (2C, Ar-C), $129.8\left(2 \mathrm{C}, \mathrm{C}_{6} \mathrm{H}_{5}-\mathrm{C}\right), 130.6(2 \mathrm{C}, \mathrm{Ar}-\mathrm{C}), 136.4(1 \mathrm{C}, \mathrm{Ar}-\mathrm{C}), 143.2\left(1 \mathrm{C}, \mathrm{C}_{6} \mathrm{H}_{5}-\mathrm{C}\right), 156.9\left(1 \mathrm{C}, \mathrm{C}_{5}\right)$, $173.8\left(1 \mathrm{C}, \mathrm{C}=\mathrm{O}\right.$ ). Anal. Calcd. for $\mathrm{C}_{33} \mathrm{H}_{47} \mathrm{ClN}_{2} \mathrm{O}_{2} ; \mathrm{C}, 73.51 ; \mathrm{H}, 8.79 ; \mathrm{N}, 5.20 \%$; Found: $\mathrm{C}, 73.43 ; \mathrm{H}, 8.69 ; \mathrm{N}$, $5.15 \%$

4.7 Synthesis of 8-[5-(4-Bromophenyl)-4-octyl-2-phenyl-3,4-dihydro-2H-pyrazol-3-yl]-octanoic acid ethyl ester (3g): Obtained from 4-Bromobenzaldehyde phenylhydrazone $2 \mathrm{~g}(5 \mathrm{mmol})$, ethyl oleate 1 (5 mmol) and chloramine-T trihydrate $(5.5 \mathrm{mmol})$ in ethyl alcohol $(20 \mathrm{~mL})$ as an oil in $60 \%$ yield. ${ }^{1} \mathrm{H} \mathrm{NMR} \mathrm{CDCl} 3: \delta 0.94(\mathrm{t}$, $\left.3 \mathrm{H}, \mathrm{CH}_{3}\right), 1.25$ (s, $\left.18 \mathrm{H}, \mathrm{CH}_{2}\right), 1.30\left(\mathrm{t}, 3 \mathrm{H}, \mathrm{CH}_{3}\right), 1.34\left(\mathrm{~s}, 2 \mathrm{H}, \mathrm{CH}_{2}\right), 1.39$ (s, $\left.2 \mathrm{H}, \mathrm{CH}_{2}\right), 1.49\left(\mathrm{~s}, 2 \mathrm{H}, \mathrm{CH}_{2}\right), 1.63$ (s, $\left.2 \mathrm{H}, \mathrm{CH}_{2}\right), 2.26\left(\mathrm{~s}, 2 \mathrm{H}, \mathrm{CH}_{2}\right), 4.10\left(\mathrm{dd}, 1 \mathrm{H}, \mathrm{C}_{3}-\mathrm{H}, J=8.6 \mathrm{~Hz}\right), 4.26\left(\mathrm{~s}, 2 \mathrm{H}, \mathrm{CH}_{2}\right), 5.16\left(\mathrm{dd}, 1 \mathrm{H}, \mathrm{C}_{4}-\mathrm{H}, J=8.2 \mathrm{~Hz}\right.$ ), $6.46\left(\mathrm{~s}, 2 \mathrm{H}, \mathrm{C}_{6} \mathrm{H}_{5}-\mathrm{H}\right), 6.60\left(\mathrm{~s}, 1 \mathrm{H}, \mathrm{C}_{6} \mathrm{H}_{5}-\mathrm{H}\right), 7.10\left(\mathrm{~s}, 2 \mathrm{H}, \mathrm{C}_{6} \mathrm{H}_{5}-\mathrm{H}\right), 7.48(\mathrm{~s}, 4 \mathrm{H}, \mathrm{Ar}-\mathrm{H}) .{ }^{13} \mathrm{C} \mathrm{NMR} \mathrm{CDCl} \mathrm{N}_{3}: \delta 13.6$ $\left(1 \mathrm{C}, \underline{\mathrm{CH}}_{3}\right), 14.3\left(1 \mathrm{C}, \underline{\mathrm{CH}}_{3}\right), 23.4\left(1 \mathrm{C}, \underline{\mathrm{CH}}_{2}\right), 24.1\left(1 \mathrm{C}, \underline{\mathrm{CH}}_{2}\right), 25.8\left(1 \mathrm{C}, \underline{\mathrm{CH}}_{2}\right), 26.6\left(1 \mathrm{C}, \underline{\mathrm{CH}}_{2}\right), 28.2\left(1 \mathrm{C}, \underline{\mathrm{CH}}_{2}\right)$, $30.4\left(3 \mathrm{C}, \underline{\mathrm{CH}}_{2}\right), 30.9\left(3 \mathrm{C}, \underline{\mathrm{C}} \mathrm{H}_{2}\right), 32.8\left(1 \mathrm{C}, \underline{\mathrm{CH}}_{2}\right), 33.8\left(1 \mathrm{C}, \underline{\mathrm{CH}}_{2}\right), 34.5\left(1 \mathrm{C}, \underline{\mathrm{CH}}_{2}\right), 44.1\left(1 \mathrm{C}, \underline{\mathrm{C}}_{4}\right), 53.2\left(1 \mathrm{C}, \underline{\mathrm{C}}_{3}\right)$, 
Synthesis of 8-(5-Aryl-4-Octyl-2-Phenyl-3,4-Dihydro-2H-Pyrazol-3-yl)-Octanoic acid ethyl esters Via $59.6\left(1 \mathrm{C}, \mathrm{OCH}_{2}\right), 113.8\left(2 \mathrm{C}, \mathrm{C}_{6} \mathrm{H}_{5}-\underline{\mathrm{C}}\right), 116.4\left(1 \mathrm{C}, \mathrm{C}_{6} \mathrm{H}_{5}-\underline{\mathrm{C}}\right), 125.5(1 \mathrm{C}, \mathrm{Ar}-\underline{\mathrm{C}}), 129.4\left(2 \mathrm{C}, \mathrm{C}_{6} \mathrm{H}_{5}-\underline{\mathrm{C}}\right), 130.4(1 \mathrm{C}$, $\operatorname{Ar}-\underline{\mathrm{C}}), 131.2(2 \mathrm{C}, \mathrm{Ar}-\underline{\mathrm{C}}), 131.8(2 \mathrm{C}, \mathrm{Ar}-\underline{\mathrm{C}}), 143.8\left(1 \mathrm{C}, \mathrm{C}_{6} \mathrm{H}_{5}-\underline{\mathrm{C}}\right), 156.4\left(1 \mathrm{C}, \underline{\mathrm{C}_{5}}\right), 173.2(1 \mathrm{C}, \underline{\mathrm{C}}=\mathrm{O})$. Anal. Calcd. For $\mathrm{C}_{33} \mathrm{H}_{47} \mathrm{BrN}_{2} \mathrm{O}_{2} ; \mathrm{C}, 67.91 ; \mathrm{H}, 8.12 ; \mathrm{N}, 4.80 \%$; Found: C, 67.83; H, 8.06; N, 4.72\%.

4.8 Synthesis of 8-[5-(4-Nitrophenyl)-4-octyl-2-phenyl-3,4-dihydro-2H-pyrazol-3-yl]-octanoic acid ethyl ester (3h): Obtained from 4-Nitrobenzaldehyde phenylhydrazone $2 \mathrm{~h}(5 \mathrm{mmol})$, ethyl oleate 1 (5 mmol) and chloramine-T trihydrate $(5.5 \mathrm{mmol})$ in ethyl alcohol $(20 \mathrm{~mL})$ as thick oil in $48 \%$ yield. ${ }^{1}{ }^{1} \mathrm{NMR} \mathrm{CDCl}_{3}: \delta 0.98$ (t, $\left.3 \mathrm{H}, \mathrm{CH}_{3}\right), 1.20\left(\mathrm{~s}, 18 \mathrm{H}, \mathrm{CH}_{2}\right), 1.28\left(\mathrm{t}, 3 \mathrm{H}, \mathrm{CH}_{3}\right), 1.36\left(\mathrm{~s}, 2 \mathrm{H}, \mathrm{CH}_{2}\right), 1.43\left(\mathrm{~s}, 2 \mathrm{H}, \mathrm{CH}_{2}\right), 1.52\left(\mathrm{~s}, 2 \mathrm{H}, \mathrm{CH}_{2}\right), 1.68$ $\left(\mathrm{s}, 2 \mathrm{H}, \mathrm{CH}_{2}\right), 2.28\left(\mathrm{~s}, 2 \mathrm{H}, \mathrm{CH}_{2}\right), 4.14\left(\mathrm{dd}, 1 \mathrm{H}, \mathrm{C}_{3}-\mathrm{H}, J=8.6 \mathrm{~Hz}\right), 4.26\left(\mathrm{~s}, 2 \mathrm{H}, \mathrm{CH}_{2}\right), 5.14\left(\mathrm{dd}, 1 \mathrm{H}, \mathrm{C}_{4}-\mathrm{H}, J=8.2\right.$ $H z), 6.40\left(\mathrm{~s}, 2 \mathrm{H}, \mathrm{C}_{6} \mathrm{H}_{5}-\mathrm{H}\right), 6.62\left(\mathrm{~s}, 1 \mathrm{H}, \mathrm{C}_{6} \mathrm{H}_{5}-\mathrm{H}\right), 7.08\left(\mathrm{~s}, 2 \mathrm{H}, \mathrm{C}_{6} \mathrm{H}_{5}-\mathrm{H}\right), 7.38(\mathrm{~s}, 4 \mathrm{H}, \mathrm{Ar}-\mathrm{H})$. Anal. Calcd. For $\mathrm{C}_{33} \mathrm{H}_{47} \mathrm{~N}_{3} \mathrm{O}_{4} ; \mathrm{C}, 72.10 ; \mathrm{H}, 8.62 ; \mathrm{N}, 7.64 \%$; Found: $\mathrm{C}, 72.01 ; \mathrm{H}, 8.59 ; \mathrm{N}, 7.55 \%$.

4.9 Synthesis of 8-[5-Furan-2-oyl-4-octyl-2-phenyl-3,4-dihydro-2H-pyrazol-3-yl]-octanoic acid ethyl ester (3i): Obtained from 2-Furanaldehyde phenylhydrazone $2 \mathbf{i}(5 \mathrm{mmol})$, ethyl oleate $\mathbf{1}(5 \mathrm{mmol})$ and chloramine-T trihydrate $(5.5 \mathrm{mmol})$ in ethyl alcohol $(20 \mathrm{~mL})$ as block solid in $68 \%$ yield. ${ }^{1} \mathrm{H} \mathrm{NMR} \mathrm{CDCl}_{3}: \delta 0.98\left(\mathrm{t}, 3 \mathrm{H}, \mathrm{CH}_{3}\right)$, 1.26 (s, $\left.18 \mathrm{H}, \mathrm{CH}_{2}\right), 1.29$ (t, $\left.3 \mathrm{H}, \mathrm{CH}_{3}\right), 1.34\left(\mathrm{~s}, 2 \mathrm{H}, \mathrm{CH}_{2}\right), 1.38\left(\mathrm{~s}, 2 \mathrm{H}, \mathrm{CH}_{2}\right), 1.48\left(\mathrm{~s}, 2 \mathrm{H}, \mathrm{CH}_{2}\right), 1.68\left(\mathrm{~s}, 2 \mathrm{H}, \mathrm{CH}_{2}\right)$, $2.34\left(\mathrm{~s}, 2 \mathrm{H}, \mathrm{CH}_{2}\right), 3.90\left(\mathrm{dd}, 1 \mathrm{H}, \mathrm{C}_{3}-\mathrm{H}, J=8.6 \mathrm{~Hz}\right.$ ), $4.34\left(\mathrm{~s}, 2 \mathrm{H}, \mathrm{CH}_{2}\right), 5.12\left(\mathrm{dd}, 1 \mathrm{H}, \mathrm{C}_{4}-\mathrm{H}, J=8.2 \mathrm{~Hz}\right), 6.36(\mathrm{~s}, 2 \mathrm{H}$, Furanoyl-H), $6.62\left(\mathrm{~s}, 2 \mathrm{H}, \mathrm{C}_{6} \mathrm{H}_{5}-\mathrm{H}\right), 6.74\left(\mathrm{~s}, 1 \mathrm{H}, \mathrm{C}_{6} \mathrm{H}_{5}-\mathrm{H}\right), 7.12\left(\mathrm{~s}, 2 \mathrm{H}, \mathrm{C}_{6} \mathrm{H}_{5}-\mathrm{H}\right), 7.52\left(\mathrm{~s}, 1 \mathrm{H}\right.$, Furanoyl-H) ${ }^{13} \mathrm{C}$ NMR $\mathrm{CDCl}_{3}: \delta 13.0\left(1 \mathrm{C}, \underline{\mathrm{CH}_{3}}\right), 14.1\left(1 \mathrm{C}, \underline{\mathrm{CH}}_{3}\right), 23.3\left(1 \mathrm{C}, \underline{\mathrm{CH}}_{2}\right), 24.5\left(1 \mathrm{C}, \underline{\mathrm{C}} \mathrm{H}_{2}\right), 25.3\left(1 \mathrm{C}, \underline{\mathrm{CH}}_{2}\right), 26.6(1 \mathrm{C}$, $\left.\mathrm{CH}_{2}\right), 28.2\left(1 \mathrm{C}, \mathrm{CH}_{2}\right), 30.4\left(3 \mathrm{C}, \mathrm{CH}_{2}\right), 31.1\left(3 \mathrm{C}, \mathrm{CH}_{2}\right), 32.8\left(1 \mathrm{C}, \mathrm{CH}_{2}\right), 34.0\left(1 \mathrm{C}, \mathrm{CH}_{2}\right), 34.6\left(1 \mathrm{C}, \mathrm{CH}_{2}\right), 44.3$ $\left(1 \mathrm{C}, \underline{\mathrm{C}}_{4}\right), 53.2\left(1 \mathrm{C}, \underline{\mathrm{C}}_{3}\right), 59.5\left(1 \mathrm{C}, \mathrm{OCH}_{2}\right), 113.0\left(2 \mathrm{C}, \mathrm{C}_{6} \mathrm{H}_{5}-\underline{\mathrm{C}}\right), 110.4$ (2C, Furanoyl-C), $115.9\left(1 \mathrm{C}, \mathrm{C}_{6} \mathrm{H}_{5}-\underline{\mathrm{C}}\right)$, 143.8 (2C, Furanoyl- $\underline{\mathrm{C}}), 128.8\left(2 \mathrm{C}, \mathrm{C}_{6} \mathrm{H}_{5}-\mathrm{C}\right), 143.5\left(1 \mathrm{C}, \mathrm{C}_{6} \mathrm{H}_{5}-\mathrm{C}\right), 156.1\left(1 \mathrm{C}, \mathrm{C}_{5}\right), 173.6(1 \mathrm{C}, \underline{\mathrm{C}}=\mathrm{O})$. Anal. Calcd. For $\mathrm{C}_{31} \mathrm{H}_{46} \mathrm{~N}_{2} \mathrm{O}_{3} ; \mathrm{C}, 75.26 ; \mathrm{H}, 9.37 ; \mathrm{N}, 5.66 \%$; Found: C, 78.53; H, 9.59; N, 5.55\%.

\section{Conclusion}

The naturally occurring precursor oleic acid derivative ethyl oleate was successfully employed in organic synthesis. This may leads to a lot of interest in biochemists and pharmacologists work in this area to study the biological potency of such compounds.

\section{Acknowledgements}

The authors are grateful to the University Grants Commission, New Delhi, for the Financial Support.

\section{References:}

[1] I. Damljanovic, M. Vukicevic, N. Radulovic, R. Palic, E. Ellmerer, Z. Ratkovic, M.D. Joksovic, R.D. Vukicevic, "Synthesis and antimicrobial activity of some new pyrazole derivatives containing ferrocene unit”, Bioorg. Med. Chem. Lett., 19, $2009,1093-1096$.

[2] Vijay V. Dabholkar, Faisal Y. Ansari, "Synthesis and characterization of selected fused isoxazole and pyrazole derivatives and their antimicrobial activity", J. Serb. Chem. Soc., 74(11), 2009, 1219-1228.

[3] E. Abdu Musad, R. Mohamed, B.A. Saeed, B.S. Vishwanath, K.M.L. Rai, "Synthesis and evaluation of antioxidant and antibacterial activities of new substituted bis(1,3,4-oxadiazoles), 3,5-bis(substituted) pyrazoles and isoxazoles", Bioorg. and Med. Chem. Letters, 21(12), 2011, 3536-3540.

[4] Ahmed I. Hashem, Ahmed S. A. Youssef, Kamal A. Kandeel, Wael S. I. Abou-Elmagd, "Conversion of some 2(3H)-furanones bearing a pyrazolyl group into other heterocyclic systems with a study of their antiviral activity”, Eur. J. Med. Chem., 42(7), 2007, 934-939.

[5] P.T. Chovatia, J.D. Akabari, P.K. Kachhadia, P.D. Zalawadia, H.S. Joshi, "Synthesis and selective antitubercular and antimicrobial inhibitory activity of 1-acetyl-3,5-diphenyl-4,5-dihydro-(1H)-pyrazole derivatives", J. Serb. Chem. Soc., 71(7), 2007, 713-720.

[6] Pedro E. Almeida da Silva, Daniela F. Ramos, Helio G. Bonacorso, Augustina I. de la Iglesia, Marli R. Oliveira, Tatiane Coelho, Jussara Navarini, Hector R. Morbidoni, Nilo Zanatta, A.P. Martins, "Synthesis and in vitro antimycobacterial activity of 3substituted 5-hydroxy-5-trifluoro[chloro]methyl]-4,5-dihydro-1H-1-(isonicotinoyl) pyrazoles", Int. J. of Antimicrobial Agents, 32, 2008, 139-144.

[7] Ashraf Hassan Abadi, Amal Abdel Haleem Eissa, Ghaneya Sayed Hassan, "Synthesis of novel 1,3,4-trisubstituted pyrazole derivatives and their evaluation as antitumor and antiangiogenic agents", Chem. Pharm. Bull., 51(7), 2003, 838-844.

[8] Adnan A. Bekhit, Tarek Abdel-Aziem, "Design, synthesis and biological evaluation of some pyrazole derivatives as antiinflammatory-antimicrobial agents", Bioorg. Med. Chem., 12, 2004, 1935-1945.

[9] N. Satheesha Rai, Balakrishna Kalluraya, B. Lingappa, Shaliny Shenoy, Vedavathi G Puranic, "Convenient access to 1,3,4trisubstituted pyrazoles carrying 5-nitrothiophene moiety via 1,3-dipolar cycloaddition of sydnones with acetylenic ketones and their antimicrobial evaluation", Eur. J. of Med. Chem., 43, 2008, 1715-1720.

[10] Rolf Huisgen, Michael Seidel, Gunter Wallbillich, Hans Knufper, Diphenyl-nitrilimin and seine 1,3-dipolaren additionen an alkene and alkine", Tetrahedron, 17, 1962, 3-29.

[11] .A.F. Gladstone, J.B. Aylward, R.O.C. Norman, "Reactions of lead tetra-acetate. Part XVIII. Oxidation of aldehyde hydrazones: a new method for the generation of nitrilimines", J. Chem. Soc. C, 1969, 2587-2598.

[12] K.B. Umesha, K.M. Lokanatha Rai and K. Ajay Kumar, "A new approach to the synthesis of pyrazoles via 1,3-dipolar cycloaddition of nitrile imines with acetyl acetone", Indian J. of Chemistry, 41B, 2002, 1450-1453.

[13] K.B. Umesha, K.M.L. Rai, M.A. Harish Nayaka, "Antioxidant and Antimicrobial Activity of 5-methyl-2-(5-methyl-1,3-diphenyl1H-pyrazole-4-carbonyl)-2,4-dihydro-pyrazol-3-one”, Int. J. of Biomed. Sci., 5(4), 2009, 359-368.

[14] B. Jayashankara, K.M. Lokanatha Rai, "Synthesis and antimicrobial studies of new series of pyrazoline bearing bis heterocycles via 1,3-dipolar cycloaddition reactions", E-Journal of Chemistry, 5(2), 2008, 309-315. 